\title{
Article \\ Did School Meal Programs and SNAP Participation Improve Diet Quality of US Children from Low-Income Households: Evidence from NHANES 2013-2014?
}

\author{
Tzuan A. Chen ${ }^{1,2, *(\mathbb{D})}$, Lorraine R. Reitzel ${ }^{1,2} \mathbb{D}$, Ezemenari M. Obasi ${ }^{1,2}$ and Jayna M. Dave ${ }^{3}$ \\ 1 Department of Psychological, Health and Learning Sciences, College of Education, The University of \\ Houston, 3657 Cullen Blvd. Stephen Power Farish Hall, Houston, TX 77204, USA; \\ lrreitze@central.uh.edu (L.R.R.); emobasi@central.uh.edu (E.M.O.) \\ 2 HEALTH Research Institute, The University of Houston, 4849 Calhoun Rd., Houston, TX 77204, USA \\ 3 USDA/ARS Children's Nutrition Research Center, Department of Pediatrics, Baylor College of Medicine, \\ 1100 Bates St., Houston, TX 77030, USA; jmdave@bcm.edu \\ * Correspondence: tchen3@central.uh.edu; Tel.: +1-713-743-6345
}

check for updates

Citation: Chen, T.A.; Reitzel, L.R.; Obasi, E.M.; Dave, J.M. Did School Meal Programs and SNAP

Participation Improve Diet Quality of US Children from Low-Income Households: Evidence from NHANES 2013-2014?. Nutrients 2021, 13, 3574. https://doi.org/10.3390/ nu13103574

Academic Editor: Licia Iacoviello

Received: 13 September 2021

Accepted: 9 October 2021

Published: 12 October 2021

Publisher's Note: MDPI stays neutral with regard to jurisdictional claims in published maps and institutional affiliations.

Copyright: (c) 2021 by the authors. Licensee MDPI, Basel, Switzerland. This article is an open access article distributed under the terms and conditions of the Creative Commons Attribution (CC BY) license (https:// creativecommons.org/licenses/by/ $4.0 /)$.

\begin{abstract}
Nutrition assistance programs such as school meals and the Supplemental Nutrition Assistance Program (SNAP) are designed to provide a safety net for the dietary intake of children from low-income families. However, compared with eligible non-participants, the relationship of diet quality with school meals only and school meals + SNAP is not well understood. The objectives of the study include: (1) To explore whether and to what extent nutrition assistance program participation (school meals only and school meals + SNAP) is related to diet quality; and (2) to examine the differences of diet quality between participating in school meals only, school meals + SNAP, or non-participation among American children. Children aged 5 to 18 years old from income eligible households who participated in the 2013-2014 National Health and Nutrition Examination Survey (NHANES) were included in this cross-sectional study $(n=1425)$. Diet quality was measured using the Healthy Eating Index (HEI)-2015 and its 13 subcomponents. A Rao-Scott Chi-square test, propensity scores approach, and Analysis of Covariance were performed. Covariates included age, sex, race/ethnicity, weight status, and family monthly poverty index. SAS survey procedures were used to incorporate the appropriate sample design weights. Participation in school meals + SNAP was not associated with higher diet quality compared to eligible non-participants or school meals-only participants. Participation in school meals + SNAP improved the intake of total dairy, but not added sugars or total vegetables compared to school meals only. Overall, school meal + SNAP participation did not significantly improve the overall diet quality of children in low-income households relative to comparable non-participants.
\end{abstract}

Keywords: National Health and Nutrition Examination Survey (NHANES); Healthy Eating Index (HEI); school meals; Supplemental Nutrition Assistance Program (SNAP); children

\section{Introduction}

Diet plays an important part in health [1,2]. Poor diet quality (e.g., high fats, added sugars, and low fiber) is a leading cause for chronic diseases like type 2 diabetes, cardiovascular disease, and some cancers such as breast cancer and colorectal cancer [3-5]. Furthermore, poor diet quality ranks among the top risk factors for chronic disease and mortality in the United States (US) [6]. The Healthy Eating Index-2015 was reported to be poor, just about 61-51 (out of 100) for US children (age 2-18), decreasing with age [7], implying poor adherence to US Dietary Guidelines among US children. Evidence showed that poor diet quality contributed to elevated disease risk among children such as hormonerelated cancers and insulin resistance [8,9], and worse school performance [10]. In addition, children having poor diet quality are more susceptible to adverse health outcomes throughout life, as dietary behaviors developed in early life often carry into adulthood $[9,11,12]$. 
Multiple determinants, such as food preferences, health beliefs [13-15], socioeconomic status (SES) [16,17], and psychosocial precursors (e.g., lack of meal planning/shopping list) [18] are implicated in the increased rate of poor diet quality among the US population. SES is a complex construct, with years of education attainment and race/ethnicity as possible indicators [16,17]. A growing body of evidence suggests that SES and dietary intake are related, such that individuals from low-income families tend to have less capacity to adhere to the dietary guidelines due to limited access to healthy foods $[16,17]$. However, fewer differences in the adherence to dietary recommendations by income level were observed among children [17]. The household and school food environments, in particular, have a great influence on youths' diet quality and behavior [19]. Diet during childhood is an important predictor of diet quality in adulthood [20], and thus, subsequent risk for dietrelated morbidity. Thus, understanding the influences of adverse factors on the healthy dietary behavior of children is imperative, especially for those from low-income families who may have limited access and resources to secure healthy food.

Examination of a population's dietary quality is critical because it provides guideline for public health policy. Individuals consume meals consisting of different foods that contain a combination of nutrients. Due to the complexity of dietary patterns, a composite measure summarizing the combinations of dietary components has been used to assess diet quality. One of the most used pre-existing diet quality indices in the US is the Healthy Eating Index (HEI) [21,22]. Poor diet quality is associated with elevated health risks that can impact costs of diet-related illness, such as diabetes, heart disease, and colorectal cancer-sizeable costs that are, in many cases, absorbed by the public [3,23,24]. Among children, there are many factors that may influence diet quality [19,25]. For example, one of the most important influences on the diet quality of children from low-income families is their participation in a nutrition assistance program [26-28].

Low-income households or individuals often rely on federal and community food and nutrition assistance programs to meet their dietary needs, and it is not unusual for eligible households to participate in more than one program [29]. Two of the largest federal nutrition assistance programs administered by the Food and Nutrition Service (FNS) of the US Department of Agriculture (USDA) are the Supplemental Nutrition Assistance Program (SNAP, formerly the Food Stamp Program) and the School Meal Programs including National School Lunch Program (NSLP) and School Breakfast Program (SBP) (hereafter, referred to as school meals).

SNAP is the largest federal food and nutrition assistance program through which eligible low-income households receive monthly benefits to purchase food at authorized retailers. In 2018, SNAP offered nutrition assistance to about 40.3 million individuals (20.1 million households) and provided an average monthly benefit of $\$ 126$ per person (\$254 per household) [30]. School meals provide meals for free or at a reduced price to children who are eligible, depending on family income guidelines. In 2019, about 29.6 million school children ate a NSLP meal each day, of which $74.1 \%$ were free or reducedprice meals [31], and 14.8 million children received a SBP meal each day, of which $85.1 \%$ were free or reduced-price meals [32]. All school meals are required to meet federal nutrition standards that align with the Dietary Guidelines for Americans (DGA) [33]. These school meals contribute a large portion of energy and nutrition for these children in low-income households [34].

Research assessing the relationship between nutrition assistance programs and diet quality have found mixed results $[26,31,33]$. For example, in one study, SNAP participants did not differ from low-income non-participants in daily caloric, macronutrient, and micronutrient intake [26]. However, SNAP participation increased certain HEI subcomponents scores (e.g., whole fruit) in adults [26,31], but not in children, as compared with low-income non-participants [26]. This is surprising as school meal programs, including the NSLP and the SBP, play an important role in children's diets and can represent up to $58 \%$ of their daily food intake [34]. Additionally, the effects of SNAP participation on sugar-sweetened beverage consumption are also inconsistent [26]. 
Several studies have examined the relationship of participation in school meal program(s) and/or SNAP alone and diet quality among different populations [26,35-39]. However, we are unaware of studies that have specifically addressed the relationship between school meals, and school meals + SNAP combined (hereafter referred to as "nutrition assistance program participation," for school meals, and/or school meals + SNAP combined) and diet quality. This study examined the association of nutrition assistance program participation with diet quality (as assessed by the Healthy Eating Index (HEI2015)) among US children ages 5-18, using the 2013 to 2014 National Health and Nutrition Examination Survey (NHANES) data. The primary objectives were to identify: (1) whether participating in a nutrition assistance program (i.e., school meal only, and school meal + SNAP combined) was associated with diet quality, and (2) the differences in diet quality between school meals only, school meals + SNAP, and non-participation.

\section{Materials and Methods}

\subsection{Participants}

The study employed data from the NHANES 2013-2014. Secondary analysis of publicly available NHANES data was determined to be exempt from review by the University of Houston Institutional Review Board. NHANES is a cross-sectional, nationally representative survey of the civilian non-institutionalized population of the US conducted by the National Center for Health Statistics (NCHS) of the Centers for Disease Control and Prevention (CDC). Detailed information about each survey and its sampling design can be found elsewhere [21]. Children 5- to 18-years old with household income $\leq 200 \%$ of the federal poverty level (FPL) were included in the analyses. Participants with missing information on any of the main independent or dependent variables: SNAP participation, school meals participation, and HEI-2015 and its 13 subcomponents were excluded from the analysis. The final sample included 1425 children, with children categorized into three grade levels: elementary, middle, and high school. The NHANES protocols were approved by the National Center for Health Statistics Ethics Review Board of the U.S. CDC; written informed consent from all the participants was obtained prior to data collection.

\subsection{Measures}

\subsubsection{Diet Quality—Healthy Eating Index-2015}

Diet quality was measured using the Healthy Eating Index (HEI-2015) [40], which assesses how well an individual's dietary intake aligns with the Dietary Guidelines for Americans 2015-2020 [41]. The HEI-2015 was calculated using the simple HEI scoring algorithm for day 1 of the 24-h dietary recall data from the NHANES. Recall data were collected by trained interviewers using the USDA Automated Multiple Pass Method. Participants 11 years or younger were interviewed with assistance. Only weekday dietary data were used to assess the association of the HEI-2015 with the participation of school meals only and school meals + SNAP. In addition, only the day 1 recall was used because the day 2 recall had a higher rate of non-response and because people tended to report less consumption on day 2 (under-reporting or survey fatigue). The use of day 1 recall only has been used in previous published studies [34,42-44]. Additional information on the 24-h dietary recall procedure can be found elsewhere [45].

The HEI-2015 is a composite measure of 13 dietary elements with 9 adequacy components (i.e., Total Fruits, Whole Fruits, Total Vegetables, Greens and Beans, Whole Grains, Dairy, Total Protein Foods, Seafood and Plant Proteins, and Fatty Acids) and 4 moderation components (i.e., Refined Grains, Sodium, Added Sugars, and Saturated Fats). The details of the HEI-2015 have been reported elsewhere [21,46]. Scores on each component sum to have a maximum score of 100. For the adequacy components, higher scores reflect greater intakes. For the moderation components, higher scores reflect lower intakes because lower intakes are more desirable. For all components, a higher value indicates a better-quality diet. The higher the total score, the greater the dietary alignment with the Dietary Guide- 
lines. An HEI score of $>80$ indicates a "good diet," a score of 51-80 indicates a diet that "needs improvement," and scores $<51$ reflect diet that is "poor" [46-48].

\subsubsection{Nutrition Assistance Program Participation}

SNAP participation was assessed with the question "\{Do you/Does any member of your household currently receive SNAP or Food Stamp benefits?" Household level SNAP was used because it is possible that the respondents benefited directly or indirectly from household resource sharing even though they were not eligible for nutrition assistance. Households with low SES were eligible to participate in SNAP; moreover, if there were children in the households attending schools, they were also eligible to receive free or reduced-price school meals. Nevertheless, eligible individuals/households could decide whether or not to participate in each nutrition assistance program. Four nutrition assistance programs participation categories were identified to address this issue. First, nutrition assistance program participation was categorized as no participation or any participation. Subsequently, any participation was further categorized into school meals only (as school meals participation is the majority for single program participants), and school meals + SNAP combined.

\subsubsection{Covariates}

Covariates included age, sex, race/ethnicity, weight status, and family monthly poverty index. Primary respondent's age, sex, and race/ethnicity (Non-Hispanic White, Non-Hispanic Black, Hispanic, and Other Race) were reported in the NHANES Demographic Variables and Sample Weights Module, and family monthly poverty level index was included in the NHANES Income Questionnaire Module. Children's body measurement data were collected by trained health technicians, and the Body Mass Index (BMI) was calculated using the standard formula (weight $(\mathrm{kg}) /$ height $\left(\mathrm{m}^{2}\right)$ ). Children's weight status was classified into one of the three categories based on the CDC's sex-specific 2000 BMIfor-age growth charts for the US: underweight and healthy weight (BMI $<85$ th percentile), overweight (BMI $\geq 85$ th percentile and BMI $<95$ th percentile), and obese (BMI $\geq 95$ th percentile) [49]. Due to infrequency of underweight category (2.69\%), underweight and healthy weight were collapsed. The family monthly poverty level index, an index for the ratio of self-reported monthly income to poverty, was used as SES indicator in the analysis because it theoretically provides a comparable SES measure that can be applied across grade levels. It is an economic measure used to decide whether the income level of an individual or family qualifies them for certain federal benefits and programs. For example, to be able to receive reduced-price lunch at school. Moreover, income eligibility for participation in some federal programs, including reduced-price lunch, is that family monthly poverty level index should be less than 1.85 [50].

\subsection{Statistical Analyses}

To alleviate the issues of endogeneity and measurement error in observational studies, propensity scores were used to address the potential nonrandom self-selection bias [51].

The descriptive statistics of the participants' characteristics were calculated for the three grade levels (elementary, middle, and high). The association of the participant characteristics and grade levels was tested using ANOVA or Rao-Scott Chi-square test for continuous and categorical variables, respectively. Sample-weighted data were used to account the NHNAES complex survey sampling methods such as survey non-response, and post-stratification adjustment. The descriptive statistics, ANOVA, and Rao-Scott Chisquare test were conducted using SAS 9.4 [52]. To address the potential self-selection bias among nutrition assistance program participants and eligible non-participants, propensity scores for the three grade levels were estimated using the mnps (multinomial propensity scores) function in the twang package [53-55] in R 3.5.0 [56]. The propensity score weighting method was applied to create two output datasets in which the distributions of the variables are balanced between groups. These two output datasets and groups include: 
(1) participation and non-participation, and (2) school meals only, school meals + SNAP, or non-participation. The procedure was performed using the covariates including age, sex, race/ethnicity, weight status, and family monthly poverty index as a set of potential confounding variables with potential correlations to nutrition assistance program(s) participation and diet quality. To compute the propensity scores through inverse probability of treatment weighting, these variables were used to fit a logistic regression model and a multinomial logistic regression model, using nutrition assistance program(s) participation and nutrition assistance program(s) non-participation, and school meals only, school meals + SNAP, or nutrition assistance program(s) non-participation as the outcome, respectively. The individual average treatment effect (ATE) weights were estimated through the covariates listed above, and the propensity scores were derived from the inverse probability of ATE weights. The calculated propensity scores were then incorporated into the final outcome models. The final outcome models were performed using SAS SURVEYREG procedure to account for the complex, stratified, multistage probability cluster sampling design. In the final outcome models, multiple linear regression models were used to evaluate the association between the two nutrition assistance program participation measures and the diet quality including HEI-2015 and its 13 subcomponents. The covariates included age, sex, race/ethnicity, weight status, and family monthly poverty index. The final outcome models were conducted separately for each outcome of interest (i.e., HEI-2015 and its 13 subcomponents) for the entire sample and by the three grade levels, and were performed using SAS 9.4 [52]. Statistical significance was set at $p<0.05$.

\section{Results}

Among the 1425 schoolchildren included in this study, 48.17, 31.46, and 20.36\% were elementary-, middle-, and high-school children, respectively (Table 1). The sex distribution was approximately equal (male: $49.22 \%$, female: $50.78 \%$ ), and the majority of the participants were non-Hispanic whites (41.55-45.67\%) and healthy weight $(56.52-61.31 \%)$ across each of the grade levels. The family monthly poverty level index ranged from 1.00 to 1.03 (below 1.85), implying meeting some federal assistance program eligibility and low SES among this analytic sample (Table 1).

The distribution of non-participation, participation in school meal program, and school meal program + SNAP varied by grade level (Table 1). The prevalence of the degree of the nutrition assistance program participation significantly differed by grade level (Rao-Scott $\left.\chi^{2}=40.81, p<0.001\right)$. More than one-third of schoolchildren did not participate in school meals or SNAP; high-school children had the highest non-participation rate (54.64\%). The higher the grade level, the lower rate the school meals + SNAP participation. In addition, BMI was significantly higher for more advanced grade levels/older children $(p s<0.001)$.

Table 1. Descriptive Statistics of Participants Characteristics by Grade Level.

\begin{tabular}{|c|c|c|c|c|}
\hline & All $(n=1425)$ & $\begin{array}{c}\text { Elementary School } \\
\qquad(n \ddagger=761)^{\mathrm{a}}\end{array}$ & $\begin{array}{l}\text { Middle School } \\
(n \neq=407)^{b}\end{array}$ & $\begin{array}{l}\text { High School } \\
(n \neq=257)^{c}\end{array}$ \\
\hline & \multicolumn{4}{|c|}{ Mean \pm SD or $n \ddagger(\%)$} \\
\hline $\begin{array}{l}\text { Age (years) } \\
\text { Sex }\end{array}$ & $11.56 \pm 0.16$ & $7.95 \pm 0.07$ & $13.56 \pm 0.05$ & $17 \pm 0.09$ \\
\hline Male & $715(49.22)$ & $386(48.67)$ & $204(49.52)$ & $125(50.04)$ \\
\hline Female & $710(50.78)$ & 375 (51.33) & $203(50.48)$ & $132(49.96)$ \\
\hline Race/Ethnicity & & & & \\
\hline Hispanic & $502(28.25)$ & $246(27.51)$ & $160(29.71)$ & $96(27.77)$ \\
\hline Non-Hispanic White & $328(44.35)$ & $188(45.62)$ & $85(41.55)$ & $55(45.67)$ \\
\hline Non-Hispanic Black & $415(18.20)$ & $239(18.11)$ & $108(18.35)$ & 68 (18.16) \\
\hline
\end{tabular}


Table 1. Cont.

\begin{tabular}{|c|c|c|c|c|}
\hline & All $(n=1425)$ & $\begin{array}{l}\text { Elementary School } \\
\quad(n \neq=761)^{a}\end{array}$ & $\begin{array}{l}\text { Middle School } \\
(n \neq=407)^{b}\end{array}$ & $\begin{array}{l}\text { High School } \\
(n \neq=257)^{c}\end{array}$ \\
\hline & \multicolumn{4}{|c|}{ Mean \pm SD or $n \ddagger(\%)$} \\
\hline Other Race & $180(9.20)$ & $88(8.76)$ & $54(10.39)$ & $38(8.41)$ \\
\hline $\operatorname{BMI}\left(\mathrm{kg} / \mathrm{m}^{2}\right){ }^{a b * * *}, \mathrm{bc} * * *, \mathrm{ac} * * *$ & $21.49 \pm 0.20$ & $18.39 \pm 0.22$ & $23.24 \pm 0.42$ & $25.93 \pm 0.6$ \\
\hline \multicolumn{5}{|l|}{ Weight Status } \\
\hline Underweight & $35(2.69)$ & $21(2.92)$ & $6(2.06)$ & $8(3.12)$ \\
\hline Healthy Weight & $785(58.97)$ & $427(61.31)$ & $220(56.52)$ & $138(57.33)$ \\
\hline Overweight & $242(17.82)$ & $127(17.56)$ & $72(19.98)$ & $43(15.15)$ \\
\hline Obese & $297(20.52)$ & $144(18.22)$ & $92(21.45)$ & $61(24.40)$ \\
\hline Family Monthly Poverty Level Index & $1.01 \pm 0.04$ & $1.02 \pm 0.04$ & $1.00 \pm 0.05$ & $1.03 \pm 0.08$ \\
\hline \multicolumn{5}{|l|}{ Family Monthly Poverty Level Category } \\
\hline Monthly Poverty Level Index $\leq 1.85$ & $1223(89.03)$ & $663(90.65)$ & $347(88.88)$ & $213(85.59)$ \\
\hline Monthly Poverty Level Index $>1.85$ & $100(10.97)$ & $44(9.35)$ & $34(11.12)$ & $22(14.41)$ \\
\hline \multicolumn{5}{|l|}{ Nutrition Assistance Program Participation *** } \\
\hline No Participation & $379(35.63)$ & $157(29.15)$ & $100(33.24)$ & $122(54.64)$ \\
\hline School Meals Only & $538(34.52)$ & $293(35.32)$ & $156(35.99)$ & $89(30.36)$ \\
\hline School Meals + SNAP & $508(29.85)$ & $311(35.53)$ & $151(30.77)$ & $46(15.00)$ \\
\hline
\end{tabular}

Note: ANOVA and Rao-Scott Chi-square test were conducted to examine the participant characteristics differences among grade levels. ${ }^{\ddagger}$ : unweighted sample size; ***: $p<0.001$; BMI: body mass index; Mean and \% are weighted using National Health and Nutrition Examination Survey exam weights. a, b, and c represent elementary school, middle school, and high school groups, respectively. ab: No Participation vs. School Meals Only; bc: School Meals Only vs. School Meals and SNAP; ac: No Participation vs. School Meals and SNAP.

\section{Comparison between No Participation vs. Any Participation}

The results showed that nutrition assistance program(s) participants had lower whole grains $(4.26 \pm 1.67$ vs. $4.97 \pm 1.71, p=0.038)$ and higher total diary scores $(7.22 \pm 1.17$ vs. $6.21 \pm 1.19, p=0.017)$ compared with eligible non-participants. There were significant differences in some of the HEI-2015 component scores by grade levels. Nutrition assistance program participants showed higher total dairy score $(3.67 \pm 5.00$ vs. $2.22 \pm 4.99$, $p=0.037)$, but lower total fruits $(-1.25 \pm 3.75$ vs. $-0.12 \pm 3.66, p=0.026)$, whole fruits $(-4.05 \pm 3.76$ vs. $-2.54 \pm 3.75, p=0.01)$, and total HEI-2015 scores $(21.45 \pm 25.54$ vs. $28.05 \pm 25.22, p=0.044)$ than non-participants in middle school children. There were no significant differences between participants and non-participants in elementary school and high school children (Table 2).

All of the groups, based on participation or grade levels, had poor diet quality (HEI2015) ranging from 42.53 to 51.06. Children who participated in school meals + SNAP had higher total dairy scores $(7.98 \pm 0.51$ vs. $6.95 \pm 0.33, p=0.049)$ and added sugars $(5.47 \pm 0.75$, vs. $4.73 \pm 0.76, p=0.007)$ than non-participants and school meals-only participants, respectively. For elementary school children, school meals + SNAP participants had lower total vegetables scores compared with school meals-only participants $(1.58 \pm 0.31$, vs. $1.96 \pm 0.36, p=0.03)$. School meals-only participants $(6.81 \pm 0.86$ vs. $4.48 \pm 0.67, p=0.045)$ and school meals + SNAP participants $(6.56 \pm 0.84$ vs. $4.48 \pm 0.67$, $p=0.029)$ consumed more sodium than eligible non-participants. School meals + SNAP participants also had higher refined grains scores than eligible non-participants $(5.69 \pm 0.74$ vs. $3.75 \pm 0.66, p=0.028)$. For middle school children, non-participants had higher total fruits scores $(2.00 \pm 0.69$, vs. $-0.11 \pm 0.60, p=0.041)$, and whole fruits scores $(2.09 \pm 0.78$, vs. $-0.23 \pm 0.50, p=0.05)$ than school meals-only participants. For high school children, there was no significant difference found in eligible non-participants, school meals-only participants, and school meals + SNAP participants (Table 3). 
Table 2. Mean Healthy Eating Index-2015 Components and Total Scores with Propensity Scores Adjusted as a Function of Any Nutrition Assistance Program Participation by Grade Level $(n=1425)$.

\begin{tabular}{|c|c|c|c|c|}
\hline & & No Participation & Any Participation & \\
\hline & & Me & & $p$-Value \\
\hline \multirow[t]{15}{*}{ All } & Energy (kilocalories) & $1825.05 \pm 361.43$ & $1821.28 \pm 358.13$ & 0.968 \\
\hline & Total Vegetables & $0.66 \pm 0.60$ & $0.69 \pm 0.60$ & 0.920 \\
\hline & Greens and Beans & $1.67 \pm 0.79$ & $1.5 \pm 0.71$ & 0.393 \\
\hline & Total Fruits & $1.69 \pm 0.87$ & $1.37 \pm 0.85$ & 0.202 \\
\hline & Whole Fruits & $1.65 \pm 0.97$ & $1.09 \pm 0.95$ & 0.079 \\
\hline & Whole Grains & $4.97 \pm 1.71$ & $4.26 \pm 1.67$ & 0.038 \\
\hline & Dairy & $6.21 \pm 1.19$ & $7.22 \pm 1.17$ & 0.017 \\
\hline & Total Protein Foods & $3.53 \pm 1.02$ & $3.36 \pm 0.97$ & 0.236 \\
\hline & $\begin{array}{l}\text { Seafood and Plant } \\
\text { Proteins }\end{array}$ & $3.14 \pm 0.79$ & $2.75 \pm 0.81$ & 0.102 \\
\hline & Fatty Acids & $2.76 \pm 1.57$ & $2.1 \pm 1.34$ & 0.250 \\
\hline & Sodium & $6.81 \pm 1.75$ & $6.82 \pm 1.70$ & 0.979 \\
\hline & Refined Grains & $4.26 \pm 1.68$ & $4.34 \pm 1.81$ & 0.816 \\
\hline & Saturated Fats & $6.54 \pm 2.10$ & $6.02 \pm 1.99$ & 0.297 \\
\hline & Added Sugars & $4.18 \pm 1.64$ & $4.22 \pm 1.63$ & 0.920 \\
\hline & HEI-2015 & $48.07 \pm 7.80$ & $45.74 \pm 7.56$ & 0.237 \\
\hline \multirow[t]{15}{*}{ Elementary School } & Energy (kilocalories) & $1031.13 \pm 289.24$ & $1005.36 \pm 258.26$ & 0.833 \\
\hline & Total Vegetables & $1.32 \pm 1.00$ & $1.34 \pm 0.91$ & 0.924 \\
\hline & Greens and Beans & $3.88 \pm 1.12$ & $3.5 \pm 1.09$ & 0.337 \\
\hline & Total Fruits & $3.32 \pm 1.29$ & $3.36 \pm 1.28$ & 0.924 \\
\hline & Whole Fruits & $3.86 \pm 1.33$ & $3.52 \pm 1.18$ & 0.519 \\
\hline & Whole Grains & $7.06 \pm 1.41$ & $5.97 \pm 1.47$ & 0.083 \\
\hline & Dairy & $4.17 \pm 1.23$ & $4.88 \pm 1.29$ & 0.253 \\
\hline & Total Protein Foods & $4.94 \pm 0.92$ & $4.92 \pm 0.82$ & 0.942 \\
\hline & $\begin{array}{l}\text { Seafood and Plant } \\
\text { Proteins }\end{array}$ & $6.82 \pm 1.03$ & $6.02 \pm 1.09$ & 0.052 \\
\hline & Fatty Acids & $5.79 \pm 1.53$ & $5.51 \pm 1.49$ & 0.685 \\
\hline & Sodium & $3.96 \pm 1.38$ & $4.34 \pm 1.43$ & 0.622 \\
\hline & Refined Grains & $4.21 \pm 2.96$ & $5.09 \pm 2.78$ & 0.221 \\
\hline & Saturated Fats & $8.16 \pm 2.06$ & $7.38 \pm 1.96$ & 0.235 \\
\hline & Added Sugars & $7.19 \pm 2.15$ & $7.29 \pm 2.08$ & 0.834 \\
\hline & HEI-2015 & $64.67 \pm 3.98$ & $63.13 \pm 4.38$ & 0.538 \\
\hline \multirow[t]{15}{*}{ Middle School } & Energy (kilocalories) & $2494.57 \pm 1819.28$ & $2451 \pm 1726.53$ & 0.809 \\
\hline & Total Vegetables & $1.51 \pm 6.33$ & $1.73 \pm 6.33$ & 0.605 \\
\hline & Greens and Beans & $6.06 \pm 3.70$ & $5.69 \pm 3.72$ & 0.069 \\
\hline & Total Fruits & $-0.12 \pm 3.66$ & $-1.25 \pm 3.75$ & 0.026 \\
\hline & Whole Fruits & $-2.54 \pm 3.75$ & $-4.05 \pm 3.76$ & 0.010 \\
\hline & Whole Grains & $3.44 \pm 6.72$ & $2.86 \pm 6.66$ & 0.298 \\
\hline & Dairy & $2.22 \pm 4.99$ & $3.67 \pm 5.00$ & 0.037 \\
\hline & Total Protein Foods & $10.02 \pm 4.67$ & $9.6 \pm 4.72$ & 0.220 \\
\hline & $\begin{array}{l}\text { Seafood and Plant } \\
\text { Proteins }\end{array}$ & $-0.86 \pm 3.83$ & $-1.29 \pm 3.70$ & 0.400 \\
\hline & Fatty Acids & $0.75 \pm 8.23$ & $-0.1 \pm 7.95$ & 0.300 \\
\hline & Sodium & $5.46 \pm 8.59$ & $4.55 \pm 8.29$ & 0.090 \\
\hline & Refined Grains & $1.61 \pm 8.63$ & $0.66 \pm 8.57$ & 0.111 \\
\hline & Saturated Fats & $6.78 \pm 8.77$ & $5.49 \pm 8.92$ & 0.143 \\
\hline & Added Sugars & $-6.28 \pm 4.73$ & $-6.13 \pm 4.70$ & 0.824 \\
\hline & HEI-2015 & $28.05 \pm 25.22$ & $21.45 \pm 25.54$ & 0.044 \\
\hline
\end{tabular}


Table 2. Cont.

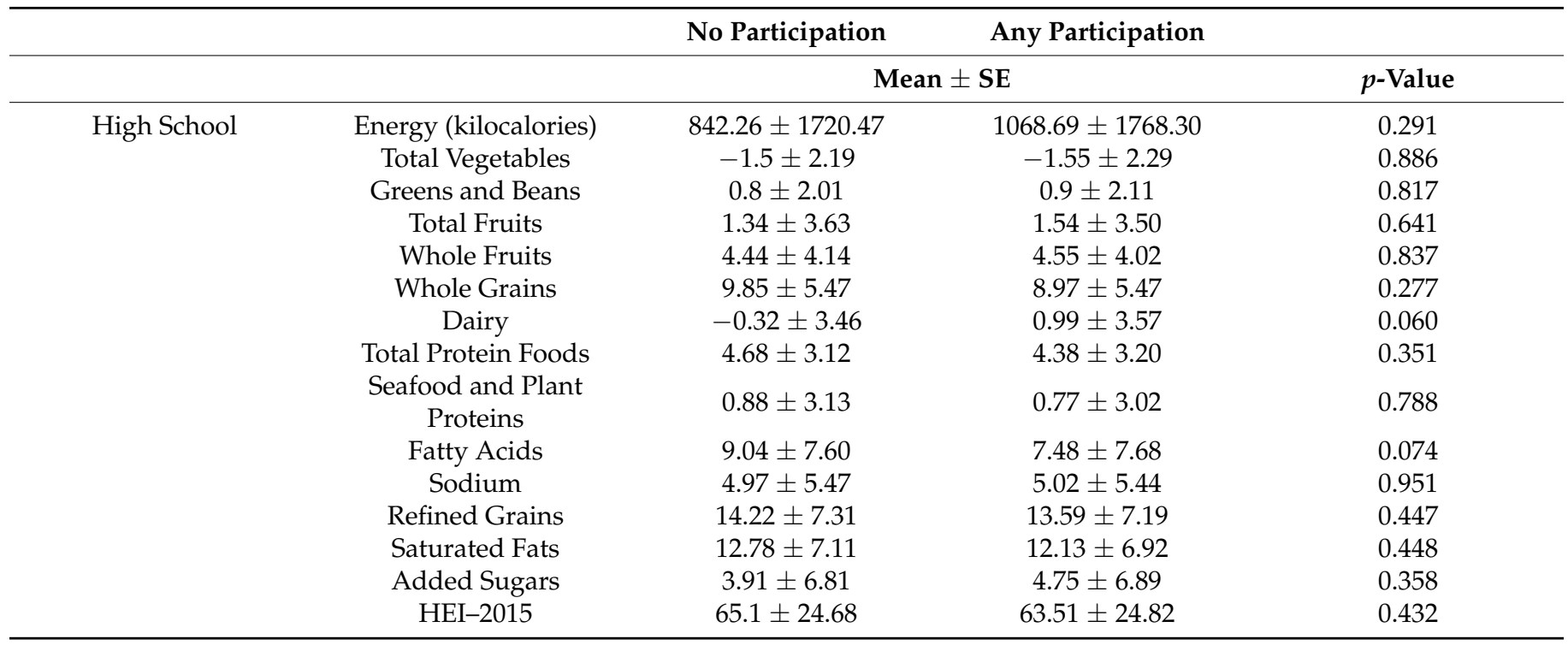

Note: The maximum score of HEI-2015 is 100. The 9 adequacy components are Total Vegetables, Greens and Beans, Total Fruits, Whole Fruits, Whole Grains, Dairy, Total Protein Foods, Seafood and Plant Proteins, and Fatty Acids; and the 4 moderation components include Sodium, Refined Grains, Saturated Fats, Added Sugars. ANOVAs were conducted to examine the Healthy Eating Index2015 components and total scores Nutrition Assistance Program Participation between Nutrition Assistance Program Participation(s). Bold values denote statistical significance at the $p<0.05$ level. 3.2. Comparison between no Participation, School Meals only, and School Meals + SNAP Combined.

Table 3. Mean Healthy Eating Index-2015 Components and Total Scores with Propensity Scores Adjusted as a Function of School Meals and SNAP Participation by Grade Level $(n=1425)$.

\begin{tabular}{|c|c|c|c|c|c|c|c|}
\hline & & $\begin{array}{c}\text { No } \\
\text { Participation a }\end{array}$ & $\begin{array}{c}\text { School } \\
\text { MealsOnly }^{b}\end{array}$ & $\begin{array}{c}\text { School Meals \& } \\
\text { SNAP c }\end{array}$ & \multirow{2}{*}{$p$-Value ${ }^{(\mathrm{ab})}$} & \multirow{2}{*}{$p$-Value ${ }^{(\text {ac) }}$} & \multirow{2}{*}{$p$-Value ${ }^{(\mathrm{bc})}$} \\
\hline \multicolumn{5}{|c|}{ Mean \pm SE } & & & \\
\hline \multirow[t]{15}{*}{ All } & Energy (kilocalories) & $1889.11 \pm 74.56$ & $1814.81 \pm 135.02$ & $1864.39 \pm 156.76$ & 0.873 & 0.585 & 0.608 \\
\hline & Total Vegetables & $1.93 \pm 0.17$ & $1.80 \pm 0.31$ & $1.74 \pm 0.28$ & 0.644 & 0.756 & 0.685 \\
\hline & Greens and Beans & $0.78 \pm 0.19$ & $1.01 \pm 0.19$ & $1.05 \pm 0.22$ & 0.336 & 0.270 & 0.839 \\
\hline & Total Fruits & $2.54 \pm 0.33$ & $2.06 \pm 0.40$ & $2.12 \pm 0.31$ & 0.234 & 0.300 & 0.818 \\
\hline & Whole Fruits & $2.43 \pm 0.38$ & $1.84 \pm 0.29$ & $1.81 \pm 0.25$ & 0.142 & 0.234 & 0.847 \\
\hline & Whole Grains & $3.61 \pm 0.43$ & $3.04 \pm 0.42$ & $3.04 \pm 0.44$ & 0.386 & 0.235 & 0.999 \\
\hline & Dairy & $6.95 \pm 0.33$ & $8.07 \pm 0.54$ & $7.98 \pm 0.51$ & 0.076 & 0.049 & 0.842 \\
\hline & Total Protein Foods & $3.42 \pm 0.19$ & $3.12 \pm 0.36$ & $3.16 \pm 0.38$ & 0.332 & 0.302 & 0.832 \\
\hline & $\begin{array}{c}\text { Seafood and Plant } \\
\text { Proteins }\end{array}$ & $1.92 \pm 0.28$ & $1.63 \pm 0.40$ & $1.77 \pm 0.41$ & 0.679 & 0.360 & 0.603 \\
\hline & Fatty Acids & $3.70 \pm 0.28$ & $2.58 \pm 0.52$ & $3.27 \pm 0.69$ & 0.608 & 0.104 & 0.193 \\
\hline & Sodium & $4.82 \pm 0.47$ & $5.82 \pm 0.45$ & $5.44 \pm 0.53$ & 0.419 & 0.146 & 0.148 \\
\hline & Refined Grains & $5.33 \pm 0.39$ & $6.16 \pm 0.41$ & $5.95 \pm 0.40$ & 0.291 & 0.170 & 0.406 \\
\hline & Saturated Fats & $5.69 \pm 0.45$ & $5.64 \pm 0.59$ & $5.81 \pm 0.72$ & 0.862 & 0.940 & 0.712 \\
\hline & Added Sugars & $5.63 \pm 0.52$ & $4.73 \pm 0.76$ & $5.47 \pm 0.75$ & 0.825 & 0.189 & 0.007 \\
\hline & HEI-2015 & $48.74 \pm 2.04$ & $47.50 \pm 1.71$ & $48.60 \pm 2.01$ & 0.964 & 0.635 & 0.463 \\
\hline \multirow[t]{8}{*}{$\begin{array}{l}\text { Elementary } \\
\text { School }\end{array}$} & Energy (kilocalories) & $1993.36 \pm 44.31$ & $1894.61 \pm 126.51$ & $1855.26 \pm 124.52$ & 0.347 & 0.536 & 0.748 \\
\hline & Total Vegetables & $2.20 \pm 0.25$ & $1.96 \pm 0.36$ & $1.58 \pm 0.31$ & 0.173 & 0.589 & 0.030 \\
\hline & Greens and Beans & $0.99 \pm 0.23$ & $1.68 \pm 0.51$ & $1.55 \pm 0.40$ & 0.210 & 0.206 & 0.536 \\
\hline & Total Fruits & $3.10 \pm 0.55$ & $2.96 \pm 0.55$ & $3.12 \pm 0.62$ & 0.987 & 0.874 & 0.697 \\
\hline & Whole Fruits & $2.93 \pm 0.64$ & $2.23 \pm 0.41$ & $2.11 \pm 0.40$ & 0.361 & 0.436 & 0.705 \\
\hline & Whole Grains & $4.01 \pm 0.46$ & $3.03 \pm 0.59$ & $3.17 \pm 0.65$ & 0.332 & 0.175 & 0.789 \\
\hline & Dairy & $7.51 \pm 0.52$ & $8.23 \pm 0.71$ & $8.1 \pm 0.49$ & 0.406 & 0.468 & 0.774 \\
\hline & Total Protein Foods & $3.49 \pm 0.29$ & $3.14 \pm 0.54$ & $2.91 \pm 0.64$ & 0.418 & 0.570 & 0.388 \\
\hline
\end{tabular}


Table 3. Cont.

\begin{tabular}{|c|c|c|c|c|c|c|c|}
\hline & & $\begin{array}{c}\text { No } \\
\text { Participation }^{\text {a }}\end{array}$ & $\begin{array}{c}\text { School } \\
\text { MealsOnly b }\end{array}$ & $\begin{array}{l}\text { School Meals \& } \\
\text { SNAP c }\end{array}$ & \multirow{2}{*}{$p$-Value ${ }^{(\mathrm{ab})}$} & \multirow{2}{*}{$p$-Value ${ }^{(\text {ac) }}$} & \multirow{2}{*}{$p$-Value ${ }^{(\mathrm{bc})}$} \\
\hline \multicolumn{5}{|c|}{ Mean \pm SE } & & & \\
\hline & $\begin{array}{c}\text { Seafood and Plant } \\
\text { Proteins }\end{array}$ & $2.35 \pm 0.33$ & $1.99 \pm 0.62$ & $2.12 \pm 0.68$ & 0.763 & 0.572 & 0.714 \\
\hline & Fatty Acids & $3.54 \pm 0.43$ & $2.40 \pm 0.74$ & $2.93 \pm 0.56$ & 0.341 & 0.242 & 0.481 \\
\hline & Sodium & $4.48 \pm 0.67$ & $6.81 \pm 0.86$ & $6.56 \pm 0.84$ & 0.045 & 0.029 & 0.574 \\
\hline & Refined Grains & $3.75 \pm 0.66$ & $6.19 \pm 0.77$ & $5.69 \pm 0.74$ & 0.087 & 0.028 & 0.313 \\
\hline & Saturated Fats & $6.67 \pm 0.76$ & $4.99 \pm 0.84$ & $4.95 \pm 0.95$ & 0.121 & 0.165 & 0.950 \\
\hline & Added Sugars & $6.04 \pm 0.64$ & $4.83 \pm 0.64$ & $4.88 \pm 0.71$ & 0.164 & 0.202 & 0.892 \\
\hline & HEI-2015 & $51.06 \pm 2.92$ & $50.44 \pm 3.44$ & $49.65 \pm 3.65$ & 0.775 & 0.901 & 0.717 \\
\hline \multirow[t]{15}{*}{$\begin{array}{l}\text { Middle } \\
\text { School }\end{array}$} & Energy (kilocalories) & $1690.78 \pm 139.89$ & $1593.19 \pm 248.52$ & $1476.52 \pm 213.00$ & 0.519 & 0.789 & 0.356 \\
\hline & Total Vegetables & $2.00 \pm 0.45$ & $2.35 \pm 0.74$ & $2.30 \pm 0.67$ & 0.743 & 0.713 & 0.894 \\
\hline & Greens and Beans & $0.81 \pm 0.37$ & $0.90 \pm 0.80$ & $0.87 \pm 0.84$ & 0.945 & 0.919 & 0.926 \\
\hline & Total Fruits & $2.00 \pm 0.69$ & $-0.11 \pm 0.60$ & $-0.16 \pm 0.50$ & 0.041 & 0.071 & 0.899 \\
\hline & Whole Fruits & $2.09 \pm 0.78$ & $-0.23 \pm 0.50$ & $-0.35 \pm 0.69$ & 0.050 & 0.050 & 0.749 \\
\hline & Whole Grains & $3.34 \pm 1.44$ & $0.87 \pm 1.50$ & $0.74 \pm 1.49$ & 0.315 & 0.334 & 0.786 \\
\hline & Dairy & $6.41 \pm 0.88$ & $7.77 \pm 0.72$ & $7.86 \pm 0.87$ & 0.364 & 0.284 & 0.896 \\
\hline & Total Protein Foods & $2.97 \pm 0.41$ & $3.32 \pm 0.74$ & $3.63 \pm 0.73$ & 0.363 & 0.647 & 0.459 \\
\hline & $\begin{array}{c}\text { Seafood and Plant } \\
\text { Proteins }\end{array}$ & $1.83 \pm 0.62$ & $0.76 \pm 0.71$ & $0.74 \pm 0.87$ & 0.390 & 0.321 & 0.966 \\
\hline & Fatty Acids & $2.92 \pm 0.67$ & $4.06 \pm 1.13$ & $4.48 \pm 1.21$ & 0.239 & 0.395 & 0.547 \\
\hline & Sodium & $4.96 \pm 0.64$ & $3.07 \pm 1.06$ & $2.94 \pm 1.27$ & 0.107 & 0.127 & 0.845 \\
\hline & Refined Grains & $6.30 \pm 1.08$ & $7.79 \pm 1.16$ & $8.04 \pm 1.05$ & 0.358 & 0.448 & 0.654 \\
\hline & Saturated Fats & $4.42 \pm 1.02$ & $5.56 \pm 1.28$ & $5.39 \pm 1.03$ & 0.539 & 0.563 & 0.810 \\
\hline & Added Sugars & $6.09 \pm 0.95$ & $4.89 \pm 1.34$ & $6.06 \pm 1.57$ & 0.984 & 0.380 & 0.085 \\
\hline & HEI-2015 & $46.15 \pm 5.87$ & $41.00 \pm 4.87$ & $42.53 \pm 5.78$ & 0.706 & 0.580 & 0.554 \\
\hline \multirow[t]{15}{*}{$\begin{array}{l}\text { High } \\
\text { School }\end{array}$} & Energy (kilocalories) & $1747.88 \pm 183.81$ & $1673.46 \pm 443.44$ & $2306.88 \pm 241.49$ & 0.061 & 0.858 & 0.069 \\
\hline & Total Vegetables & $1.69 \pm 0.26$ & $1.54 \pm 0.63$ & $1.84 \pm 0.49$ & 0.807 & 0.789 & 0.660 \\
\hline & Greens and Beans & $0.40 \pm 0.34$ & $0.86 \pm 0.61$ & $1.33 \pm 0.43$ & 0.120 & 0.511 & 0.401 \\
\hline & Total Fruits & $1.64 \pm 0.40$ & $2.31 \pm 0.72$ & $1.60 \pm 0.50$ & 0.946 & 0.346 & 0.077 \\
\hline & Whole Fruits & $1.85 \pm 0.54$ & $2.05 \pm 0.80$ & $1.81 \pm 0.59$ & 0.944 & 0.817 & 0.609 \\
\hline & Whole Grains & $3.26 \pm 0.84$ & $3.24 \pm 1.09$ & $2.31 \pm 1.05$ & 0.408 & 0.983 & 0.263 \\
\hline & Dairy & $6.83 \pm 0.58$ & $8.97 \pm 1.41$ & $8.23 \pm 1.25$ & 0.265 & 0.114 & 0.468 \\
\hline & Total Protein Foods & $3.44 \pm 0.24$ & $3.10 \pm 0.53$ & $2.84 \pm 0.37$ & 0.181 & 0.560 & 0.604 \\
\hline & $\begin{array}{l}\text { Seafood and Plant } \\
\text { Proteins }\end{array}$ & $1.00 \pm 0.46$ & $1.10 \pm 0.41$ & $1.05 \pm 0.56$ & 0.935 & 0.851 & 0.943 \\
\hline & Fatty Acids & $4.51 \pm 0.78$ & $1.89 \pm 1.26$ & $3.16 \pm 1.48$ & 0.379 & 0.063 & 0.292 \\
\hline & Sodium & $4.30 \pm 0.80$ & $4.88 \pm 1.11$ & $3.89 \pm 0.87$ & 0.682 & 0.671 & 0.279 \\
\hline & Refined Grains & $5.97 \pm 0.83$ & $5.91 \pm 0.91$ & $5.42 \pm 0.77$ & 0.637 & 0.960 & 0.407 \\
\hline & Saturated Fats & $5.78 \pm 0.99$ & $5.05 \pm 1.52$ & $5.05 \pm 1.63$ & 0.564 & 0.592 & 0.995 \\
\hline & Added Sugars & $4.72 \pm 0.91$ & $5.28 \pm 2.05$ & $6.54 \pm 1.53$ & 0.136 & 0.741 & 0.190 \\
\hline & HEI-2015 & $45.39 \pm 2.17$ & $46.17 \pm 5.24$ & $45.05 \pm 4.46$ & 0.930 & 0.883 & 0.768 \\
\hline
\end{tabular}

Note: The maximum score of HEI-2015 is 100. The 9 adequacy components are Total Vegetables, Greens and Beans, Total Fruits, Whole Fruits, Whole Grains, Dairy, Total Protein Foods, Seafood and Plant Proteins, and Fatty Acids; and the 4 moderation components include Sodium, Refined Grains, Saturated Fats, Added Sugars. ANOVAs were conducted to examine the Healthy Eating Index-2015 components and total scores Nutrition Assistance Program Participation among School Meals and SNAP Participation. Bold values denote statistical significance at the $p<0.05$ level. Letter $\mathrm{a}, \mathrm{b}$, and c represent No Participation, School Meals Only, and School Meals and SNAP, respectively. ab: No Participation vs. School Meals Only; bc: School Meals Only vs. School Meals and SNAP; ac: No Participation vs. School Meals and SNAP.

\section{Discussion}

To our knowledge, this study is the first to investigate the association between diet quality and school meal program in conjunction with SNAP participation accounting for potential self-selection. School meal eligible children can receive a school breakfast/lunch free or at a reduced price from the NSLP and/or the SBP according to income guidelines. Children in households with incomes below $130 \%$ of the poverty level or those receiving SNAP qualify for free meals, and those with family incomes between $130-185 \%$ of the poverty line qualify for reduced-price meals [50]. Most of the eligible low-income families participated in more than one nutrition assistance program, which makes it challenging to measure the specific effect of any individual nutrition assistance program [57]. 
Several important findings have emerged from this study. Energy intakes were not different between participants and eligible non-participants across all three grade levels. Compared with eligible non-participants, participating in school meal program alone or school meals + SNAP combined was associated with fewer whole grains and more dairy consumption for all grade levels combined. Overall, children participating in nutrition assistance program(s) (school meals or school meals + SNAP) had lower HEI-2015 scores and energy intake relative to non-participants, although these differences did not reach statistical significance. These findings are partially consistent with previous research in a low-income sample of children focusing on single nutrition assistance program participation, where significant differences by SNAP participation were not found in total energy, HEI-2005 and macronutrients for children aged 4 to 19 [27] or in adults [26].

Among elementary school students in the current sample, students who only participated in school meals significantly consumed more total vegetables relative to those who participated in both school meals and SNAP. Although nutrition assistance programs aim to improve program participants' dietary quality, our study showed that participation in nutrition assistance programs did not always help in reducing the intakes of HEI moderation components. Compared with eligible non-participants, the intake of sodium and refined grains was significantly higher for school meals alone participants or school meals + SNAP participants among elementary children. This finding complements and extends prior research on the effect of school meal programs on HEI moderation components. Excessive sodium and saturated fat consumption have also been shown to be associated with school meal program participation $[38,58,59]$. Those who were also receiving SNAP benefits consumed more added sugars and less total vegetables, which suggested that in addition to the school meals, household SNAP participation may only have a limited benefit to children's diet. Previous research from the first School Nutrition Dietary Assessment Study (SNDA-I) found that NSLP participation led to a higher intake of dietary fat and a lower intake of added sugars than was found amongst non-participants [58]. Findings from SNDA-III showed both NSLP and SBP participation were associated with an increase in many key nutrients such as magnesium and vitamin $\mathrm{C}$, but an increase in sodium was associated with NSLP participation alone [42].

It is notable that similar findings were found among middle school students. Our study revealed significant difference in HEI-2015 scores between non-participation and any nutrition assistance programs participation for middle school children, with nutrition assistance programs participants having significantly lower HEI-2015 relative to eligible non-participants; however, the difference of HEI-2015 scores between school meal program + SNAP participants and income-eligible nonparticipants was not significant. Previous studies have shown that around one out of five children in low-income households did not meet the dietary recommendations $[27,60]$ and SNAP participation did not improve diet quality in children in low-income households [27].

Additionally, the present study also showed that the consumptions of total fruits and whole fruits were less for nutrition assistance program(s) participants (i.e., school meals only or school meals + SNAP) than eligible non-participants in middle schools. Further segregating the effects between school meals only and school meals + SNAP, the significantly lower total fruits consumption was only found for school meals-only participants relative to eligible non-participants. Lastly, in the current sample, no significant differences in HEI-2015 and subcomponents between nutrition assistance program participation and non-participation were found among high school students.

Although no prior studies showing participation in school meals was associated with decreased fruits consumption specifically using the sample of middle/high school students, previous research has revealed that overall diet quality was equivalent for NSLP participants and non-participants [61,62]. Additionally, there also have been undesirable associations observed between school meals and dietary measures such as fat intake $[58,59]$. Moreover, previous studies using NHANES data showed that SNAP participants had significant lower consumption of most components of diet including fruits relative to 
comparable low-income non-participants $[26,63,64]$. These findings suggest that nutrition assistance program participation may not improve the nutrition of children's diet. To encourage the selection of vegetables and fruits, a prior study conducted in middle and high schools showed that school-level factors, such as a visual assessment of the quality of fruits and vegetables served and the variety of fruits and vegetables served, were associated with increased fruit and vegetable consumptions [65]. Available food resources within the home may fluctuate over time, as food stocks are usually replenished periodically [66]. Due to the variation of food stocks, the timing of the NHANES diet interview for participants may influence diet intake especially for participants from low-income households. Moreover, the use of SNAP might affect diet quality changes due to the timing of the survey, as other studies show that the SNAP cycle is relevant to the analysis [66]. Specifically, previous studies showed that SNAP participants experienced cyclic food intake with large declines near the end of the benefit month, implying the use of SNAP might affect diet quality changes due to the timing of survey or timing of the SNAP receipt [66].

There are several strengths and weaknesses of the current research that warrant discussion. The current study builds upon the extant literature regarding school-age children and nutrition assistance program participation with diet quality using the propensity score while adding to the literature by separating nutrition assistance programs' effects that were not explored previously (school meals only or school meals + SNAP). The caloric intakes and HEI-2015 scores showed no significant differences among the degree of participation in nutrition assistance programs after adjusting for covariates and propensity score, meaning the participation in nutrition assistance program(s) did not improve participants' diet quality relative to their nutrition assistance program-eligible non-participants.

The strengths of our study include the use of a large nationally representative sample of the US population to examine these associations. NHANES employs valid and reliable measure of diet, and standardized protocol for weight and height. Another strength of this study is the use of propensity scores to mitigate issues with endogeneity and self-section bias. The use of propensity scores has emerged as an approach in the estimation of a causal treatment effects in economic research. This approach is a statistical technique used in observational studies such as NHANES [67,68]; it accounts for unobserved individual differences when treatment assignment is not random. Given the nature of cross-sectional observational data like NHANES, nutrition assistance program participation might not directly affect the diet quality; yet, unobserved characteristics (e.g., health conditions) associated with nutrition assistance program participation might primarily account for the relationship with diet quality rather than participation itself. A previous study had shown that state SNAP policies and personal traits were strongly related to SNAP participation [69].

A limitation of this study was its cross-sectional nature, which does not allow causal inference. Similar to many previous studies, the results do not allow for interpretation of the causal effects of nutrition assistance program participation on diet outcomes. The lack of information on when the households were food insecure relative to when they received nutrition assistance program(s) benefits constrains the application of statistical analyses to address the self-selection bias issue on the nutrition assistance program participation [67]. Although our models adjusted for the possible confounding variables, and addressed self-selection bias with propensity scores, it is possible that unmeasured or unobserved confounding factors might affect both nutrition assistance program participation and diet quality. This may explain in part the association between nutrition assistance program participation and diet quality. Understanding of other unstudied factors, such as individual's health status, is needed to optimize efforts to improve dietary quality for children participating in nutrition assistance programs. Single time-point assessments for dietary intake may be subject to day-to-day variation and underreporting is a common problem in dietary intake data. School meals and SNAP are included in this study to account for the interaction and scope of program participation for US youth but not the Special Supplemental Nutrition Program for Women, Infants9, and Children (WIC) due to the 
small number of WIC participants in this targeted population. Like previous studies, this study included weekday data only [34,70]. In addition, bias in diet assessment methods is possible due to the use of single dietary recall, and the potential measurement errors are associated with self-reported dietary intake. Another limitation is that this study did not examine sex, race, or weight status differences; however, they are worth exploring in the future. Although this study utilized data for 2013-2014, HEI-2015 was used to assess diet quality instead of HEI-2010. However, this is not a concern since several updates were made to NSLP and SBP meal guidelines in 2012 that align with HEI-2015 [71,72].

Findings from this study call for additional research to clarify the relationship of the nutrition assistance program participation with diet quality and to determine whether nutrition assistance program participation improves diet quality. Longitudinal studies are needed to address the issue of causality and elucidate the long-term effects of how the influence of nutrition assistance program participation changes with diet quality. Ultimately, if such studies find that nutrition assistance program participation enhances overall diet quality in low-income families, the potential health implications are substantial. Our results showed that participation in SNAP did not improve children's diet quality; however, household-level SNAP participation was used in this study, so it is likely that NHANES participants did not benefit from the sources gained from other household members, as the benefit amounts might not be sufficient to smooth food intake over the benefit month for other household members. Furthermore, prior studies suggested that nutrition knowledge often associated with income and education level may partly mediate disparities in dietary intake and quality $[64,73,74]$. Therefore, the implementation of nutrition education programs alongside nutrition assistance programs is important for promoting awareness of healthy nutrition and improving health among low-income families, which could also serve as a prevention against increased disease risk in adult life [75].

\section{Conclusions}

Although nutrition assistance programs are aimed to provide a safety net for lowincome households with children, school meals + SNAP participation did not improve the overall nutritional quality of children's diets from low-income households. Participation in school meals + SNAP was not associated with better adequacy food components, higher diet quality, or lower moderation food components relative to eligible non-participants. SNAP participation along with school meals added the ameliorative effect on dairy but not added sugars and total vegetables except for within elementary school children. The current study was cross-sectional; however, the development of future longitudinal work is needed to delineate causal pathways between nutrition assistance program(s) participation and diet quality among children and adolescents to promote US youth's health. Our findings highlight the need for additional research to better understand the complex interplay between nutrition assistance program participation and diet quality for youths from low-income families.

Author Contributions: Conceptualization, T.A.C. and J.M.D.; Methodology, T.A.C. and J.M.D.; Software, T.A.C.; Validation, T.A.C. and J.M.D.; Formal analysis, T.A.C.; Writing-original draft preparation, T.A.C.; Writing—review and editing, T.A.C., L.R.R., E.M.O. and J.M.D.; Visualization, T.A.C. All authors have read and agreed to the published version of the manuscript.

Funding: Work on the manuscript was supported by the National Institute on Minority Health and Health Disparities through the University of Houston's HEALTH Center for Addictions Research and Cancer Prevention (U54MD015946 to E.M.O., in which T.A.C., E.M.O. and L.R.R. are supported). This work is also a publication of the USDA/ARS Children's Nutrition Research Center, Department of Pediatrics, Baylor College of Medicine, Houston, TX, USA. This project was supported, in part, through federal funds from the US Department of Agriculture (USDA)/Agricultural Research Service under Cooperative Agreement no. 3092-51000-058-2S (J.M.D.). Its contents are solely the responsibility of the authors and do not necessarily represent the official views of the project supporters.

Institutional Review Board Statement: Not applicable. 
Informed Consent Statement: Informed consent was obtained from all subjects involved in the NHANES study.

Data Availability Statement: The data analysis used publicly available data which can be accessed at: https: / / wwwn.cdc.gov/nchs/nhanes/continuousnhanes/default.aspx?Begin Year=2013 (accessed on 31 January 2019).

Acknowledgments: The authors wish to thank Karen Cullen for her valuable comments on this manuscript.

Conflicts of Interest: E.M.O. is the founder and sole owner of HEALTH Equity Empowerment, LLC.; authors have no additional conflicts to report.

\section{References}

1. Willett, W.C. Diet and health: What should we eat? Science 1994, 264, 532-537. [CrossRef] [PubMed]

2. McGinnis, J.M.; Foege, W.H. Actual causes of death in the United States. JAMA 1993, 270, 2207-2212. [CrossRef] [PubMed]

3. McCullough, M.L.; Feskanich, D.; Stampfer, M.J.; Giovannucci, E.L.; Rimm, E.B.; Hu, F.B.; Spiegelman, D.; Hunter, D.J.; Colditz, G.A.; Willett, W.C. Diet quality and major chronic disease risk in men and women: Moving toward improved dietary guidance. Am. J. Clin. Nutr. 2002, 76, 1261-1271. [CrossRef]

4. Potter, J.; Brown, L.; Williams, R.L.; Byles, J.; Collins, C.E. Diet Quality and Cancer Outcomes in Adults: A Systematic Review of Epidemiological Studies. Int. J. Mol. Sci. 2016, 17, 1052. [CrossRef]

5. Fung, T.T.; Hu, F.B.; McCullough, M.L.; Newby, P.K.; Willett, W.C.; Holmes, M.D. Diet Quality Is Associated with the Risk of Estrogen Receptor-Negative Breast Cancer in Postmenopausal Women. J. Nutr. 2006, 136, 466-472. [CrossRef]

6. Murray, C.J.L.; Atkinson, C.; Bhalla, K.; Birbeck, G.; Burstein, R.; Chou, D.; Dellavalle, R.; Danaei, G.; Ezzati, M.; Fahimi, A.; et al. The state of US health, 1990-2010: Burden of diseases, injuries, and risk factors. JAMA 2013, 310, 591-606. [CrossRef]

7. USDA Dietary Guidelines for Americans 2020-2025. Available online: https://www.dietaryguidelines.gov/sites/default/files / 2020-12/Dietary_Guidelines_for_Americans_2020-2025.pdf (accessed on 28 September 2021).

8. Cheng, G.; Gerlach, S.; Libuda, L.; Kranz, S.; Karaolis-Danckert, N.; Kroke, A.; Buyken, A.E.; Günther, A.L.B. Diet Quality in Childhood Is Prospectively Associated with the Timing of Puberty but Not with Body Composition at Puberty Onset. J. Nutr. 2009, 140, 95-102. [CrossRef]

9. Marshall, S.; Burrows, T.; Collins, C.E. Systematic review of diet quality indices and their associations with health-related outcomes in children and adolescents. J. Hum. Nutr. Diet. 2014, 27, 577-598. [CrossRef] [PubMed]

10. Florence, M.D.; Asbridge, M.; Veugelers, P.J. Diet Quality and Academic Performance. J. Sch. Health 2008, 78, 209-215. [CrossRef]

11. Dalwood, P.; Marshall, S.; Burrows, T.L.; McIntosh, A.; Collins, C.E. Diet quality indices and their associations with health-related outcomes in children and adolescents: An updated systematic review. Nutr. J. 2020, 19, 118. [CrossRef] [PubMed]

12. Brown, H.W.; Roberts, J. Exploring the Factors Contributing to Sibling Correlations in BMI: A Study Using the Panel Study of Income Dynamics. Obesity 2012, 20, 978-984. [CrossRef] [PubMed]

13. Leung, C.W.; Epel, E.S.; Ritchie, L.D.; Crawford, P.B.; Laraia, B.A. Food Insecurity Is Inversely Associated with Diet Quality of Lower-Income Adults. J. Acad. Nutr. Diet. 2014, 114, 1943-1953.e2. [CrossRef] [PubMed]

14. Skinner, J.D.; Carruth, B.R.; Bounds, W.; Ziegler, P.J. Children's food preferences: A longitudinal analysis. J. Am. Diet. Assoc. 2002, 102, 1638-1647. [CrossRef]

15. Norton, P.A.; Falciglia, G.A.; Ricketts, C. Motivational determinants of food preferences in adolescents and pre-adolescents. Ecol. Food Nutr. 2000, 39, 169-182. [CrossRef]

16. Wang, D.D.; Leung, C.W.; Li, Y.; Ding, E.L.; Chiuve, S.E.; Hu, F.B.; Willett, W.C. Trends in dietary quality among adults in the United States, 1999 through 2010. JAMA Intern. Med. 2014, 174, 1587-1595. [CrossRef]

17. Kirkpatrick, S.I.; Dodd, K.W.; Reedy, J.; Krebs-Smith, S.M. Income and race/ethnicity are associated with adherence to food-based dietary guidance among US adults and children. J. Acad. Nutr. Diet. 2012, 112, 624-635. [CrossRef]

18. Ranjit, N.; Macias, S.; Hoelscher, D. Factors related to poor diet quality in food insecure populations. Transl. Behav. Med. 2020, 10, 1297-1305.

19. Birch, L.L.; Davison, K.K. Family environmental factors influencing the developing behavioral controls of food intake and childhood overweight. Pediatr. Clin. 2001, 48, 893-907. [CrossRef]

20. Mikkilä, V.; Räsänen, L.; Raitakari, O.T.; Pietinen, P.; Viikari, J. Longitudinal changes in diet from childhood into adulthood with respect to risk of cardiovascular diseases: The Cardiovascular Risk in Young Finns Study. Eur. J. Clin. Nutr. 2004, 58, 1038-1045. [CrossRef]

21. Overview \& Background of the Healthy Eating Index National Cancer Institute, Division of Cancer Control \& Population Sciences. Available online: https:/ / epi.grants.cancer.gov / hei / (accessed on 9 August 2021).

22. Kennedy, E.T.; Ohls, J.; Carlson, S.; Fleming, K. The healthy eating index: Design and applications. J. Am. Diet. Assoc. 1995, 95, 1103-1108. [CrossRef] 
23. Schwingshackl, L.; Hoffmann, G. Diet Quality as Assessed by the Healthy Eating Index, the Alternate Healthy Eating Index, the Dietary Approaches to Stop Hypertension Score, and Health Outcomes: A Systematic Review and Meta-Analysis of Cohort Studies. J. Acad. Nutr. Diet. 2015, 115, 780-800.e5. [CrossRef]

24. Yusof, A.S.; Isa, Z.M.; Shah, S.A. Dietary Patterns and Risk of Colorectal Cancer: A Systematic Review of Cohort Studies (2000-2011). Asian Pac. J. Cancer Prev. 2012, 13, 4713-4717. [CrossRef]

25. Patrick, H.; Nicklas, T.A. A Review of Family and Social Determinants of Children's Eating Patterns and Diet Quality. J. Am. Coll. Nutr. 2005, 24, 83-92. [CrossRef] [PubMed]

26. Andreyeva, T.; Tripp, A.S.; Schwartz, M.B. Dietary quality of Americans by Supplemental Nutrition Assistance Program participation status: A systematic review. Am. J. Prev. Med. 2015, 49, 594-604. [CrossRef]

27. Leung, C.W.; Blumenthal, S.J.; Hoffnagle, E.E.; Jensen, H.H.; Foerster, S.B.; Nestle, M.; Cheung, L.W.; Mozaffarian, D.; Willett, W.C. Associations of Food Stamp Participation with Dietary Quality and Obesity in Children. Pediatrics 2013, 131, 463-472. [CrossRef] [PubMed]

28. Yen, S.T. The effects of SNAP and WIC programs on nutrient intakes of children. Food Policy 2010, 35, 576-583. [CrossRef]

29. Coleman-Jensen, A.; Gregory, C.; Singh, A. Household Food Security in the United States in 2013; United States Department of Agriculture: Washington, DC, USA, 2014.

30. SNAP Persons, Households, Benefits, and Average Monthly Benefit per Person \& Household: FY16 through FY19 National View Summary. Available online: https:/ / www.fns.usda.gov/pd/supplemental-nutrition-assistance-program-snap (accessed on 9 August 2021).

31. USDA Food and Nutrition Service School Lunch Program. Available online: https://fns-prod.azureedge.net/sites/default/files/ resource-files/slsummar-5.pdf (accessed on 9 August 2021).

32. USDA Food and Nutrition Service School Breakfast Program. Available online: https://fns-prod.azureedge.net/sites/default/ files/resource-files/sbsummar-5.pdf (accessed on 9 August 2021).

33. Government Accountability Office. School Lunch: Implementing Nutrition Changes Was Challenging and Clarification of Oversight Requirements Is Needed. GAO-14-104. 2014. Available online: https:/ / www.gao.gov/products/gao-14-104 (accessed on 9 August 2021).

34. Cullen, K.W.; Chen, T.-A. The contribution of the USDA school breakfast and lunch program meals to student daily dietary intake. Prev. Med. Rep. 2016, 5, 82-85. [CrossRef]

35. Gregory, C.A.; Ver Ploeg, M.; Andrews, M.; Coleman-Jensen, A. Supplemental Nutrition Assistance Program (SNAP) Participation Leads to Modest Changes in Diet Quality. (No. 1477-2017-3991). 2013. Available online: https://www.ers.usda.gov/webdocs/ publications / 45059/36939_err147.pdf?v=5272.1 (accessed on 9 August 2021).

36. Duffy, P.; Zizza, C.; Jacoby, J.; Tayie, F.A. Diet Quality is Low among Female Food Pantry Clients in Eastern Alabama. J. Nutr. Educ. Behav. 2009, 41, 414-419. [CrossRef]

37. Todd, J.E.; Ploeg, M.V. Caloric Beverage Intake Among Adult Supplemental Nutrition Assistance Program Participants. Am. J. Public Health 2014, 104, e80-e85. [CrossRef]

38. Clark, M.A.; Fox, M.K. Nutritional Quality of the Diets of US Public School Children and the Role of the School Meal Programs. J. Am. Diet. Assoc. 2009, 109, S44-S56. [CrossRef]

39. Cohen, J.F.; Richardson, S.; Parker, E.; Catalano, P.J.; Rimm, E.B. Impact of the new US Department of Agriculture school meal standards on food selection, consumption, and waste. Am. J. Prev. Med. 2014, 46, 388-394. [CrossRef]

40. Curtin, L.R.; Mohadjer, L.K.; Dohrmann, S.M.; Kruszon-Moran, D.; Mirel, L.B.; Carroll, M.D.; Hirsch, R.; Burt, V.L.; Johnson, C.L. National Health and Nutrition Examination Survey: Sample Design, 2007-2010. Vital and Health Statistics. Series 2, Data Evaluation and Methods Research; U.S. Department of Health and Human Services: Hyattsville, MD, USA, 2013; pp. 1-23.

41. You, A. Nutrition and Your Health: 2015-2020 Dietary Guidelines for Americans, 8th ed.; US Government Printing Office: Washington, DC, USA, 2015.

42. Storey, M.L.; Anderson, P.A. Vegetable Consumption and Selected Nutrient Intakes of Women of Childbearing Age. J. Nutr. Educ. Behav. 2016, 48, 691-696.e1. [CrossRef]

43. Powell, E.S.; Smith-Taillie, L.P.; Popkin, B.M. Added Sugars Intake Across the Distribution of US Children and Adult Consumers: 1977-2012. J. Acad. Nutr. Diet. 2016, 116, 1543-1550.e1. [CrossRef] [PubMed]

44. Mesirow, M.; Welsh, J.A. Changing Beverage Consumption Patterns Have Resulted in Fewer Liquid Calories in the Diets of US Children: National Health and Nutrition Examination Survey 2001-2010. J. Acad. Nutr. Diet. 2015, 115, 559-566.e4. [CrossRef] [PubMed]

45. Centers for Disease Control and Prevention: National Health and Nutrition Examination Survey (NHANES): MEC in-Person Dietary Interviewers Procedures Manual. Available online: https://www.cdc.gov/nchs/data/nhanes/nhanes_05_06/dietary_ mec.pdf (accessed on 9 August 2021).

46. Reedy, J.; Lerman, J.L.; Krebs-Smith, S.M.; Kirkpatrick, S.I.; Pannucci, T.E.; Wilson, M.M.; Subar, A.F.; Kahle, L.L.; Tooze, J.A. Evaluation of the healthy eating index-2015. J. Acad. Nutr. Diet. 2018, 118, 1622-1633. [CrossRef] [PubMed]

47. Krebs-Smith, S.M.; Pannucci, T.E.; Subar, A.F.; Kirkpatrick, S.I.; Lerman, J.L.; Tooze, J.A.; Wilson, M.M.; Reedy, J. Update of the healthy eating index: HEI-2015. J. Acad. Nutr. Diet. 2018, 118, 1591-1602. [CrossRef] [PubMed]

48. Tek, N.A.; Yildiran, H.; Akbulut, G.; Bilici, S.; Koksal, E.; Karadag, M.G.; Sanlıer, N. Evaluation of dietary quality of adolescents using Healthy Eating Index. Nutr. Res. Pract. 2011, 5, 322-328. [CrossRef] 
49. Centers for Disease Control and Prevention: Defining Childhood Weight Status BMI for Children and Teen. Available online: https:/ / www.cdc.gov/obesity/childhood/defining.html (accessed on 28 September 2021).

50. Child Nutrition Programs; Income Eligibility Guidelines: A Notice by the Food and Nutrition Service on 03/29/2013. Available online: https://www.fns.usda.gov/school-meals/fr-032913a (accessed on 9 August 2021).

51. Rosenbaum, P.R.; Rubin, D.B. The central role of the propensity score in observational studies for causal effects. Biometrika 1983, 70, 41-55. [CrossRef]

52. SAS Institute Inc. SAS (Version 9.4) [Computer Software]; SAS Institute, Inc.: Cary, NC, USA, 2014.

53. Burgette, L.; Griffin, B.A.; McCaffrey, D. Propensity Scores for Multiple Treatments: A Tutorial for the Mnps Function in the Twang Package; R Package; Rand Corporation: Santa Monica, CA, USA, 2017.

54. McCaffrey, D.F.; Griffin, B.A.; Almirall, D.; Slaughter, M.E.; Ramchand, R.; Burgette, L. A tutorial on propensity score estimation for multiple treatments using generalized boosted models. Stat. Med. 2013, 32, 3388-3414. [CrossRef]

55. Ridgeway, G.; McCaffrey, D.; Morral, A.; Burgette, L.; Griffin, B. Toolkit for Weighting and Analysis of Nonequivalent Groups: A Tutorial for the R TWANG Package; RAND Corporation: Santa Monica, CA, USA, 2014; Available online: https://www.rand.org/ pubs/tools/TL136z1.html (accessed on 9 March 2019).

56. R Core Team. R: A Language and Environment for Statistical Computing; R Foundation for Statistical Computing: Vienna, Austria, 2014.

57. Burstein, N.R. Understanding the Determinants of Supplemental Nutrition Assistance Program Participation. 2009. Available online: https:// fns-prod.azureedge.net/sites/default/files/DeterminantsSummary.pdf (accessed on 9 August 2021).

58. Gleason, P.M.; Suitor, C.W. Eating at school: How the National School Lunch Program affects children's diets. Am. J. Agric. Econ. 2003, 85, 1047-1061. [CrossRef]

59. Gordon, A.R.; Devaney, B.L. Burghardt, J.A. Dietary effects of the National School Program and the School Breaksfast Program. Am. J. Clin. Nutr. 1995, 61, 221S-231S. [CrossRef] [PubMed]

60. Nutrition and Weight Status-Healthy People. Healthy People 2010 Topics \& Objectives. Available online: https://www. healthypeople.gov/2020/topics-objectives/topic/nutrition-and-weight-status (accessed on 9 August 2021).

61. Campbell, B.L.; Nayga, R.M., Jr.; Park, J.L.; Silva, A. Does the National School Lunch Program improve children's dietary outcomes? Am. J. Agric. Econ. 2011, 93, 1099-1130. [CrossRef]

62. Bhattacharya, J.; Currie, J.; Haider, S. Poverty, food insecurity, and nutritional outcomes in children and adults. J. Health Econ. 2004, 23, 839-862. [CrossRef]

63. Wolfson, J.A.; Bleich, S.N. Fruit and vegetable consumption and food values: National patterns in the United States by Supplemental Nutrition Assistance Program eligibility and cooking frequency. Prev. Med. 2015, 76, 1-7. [CrossRef] [PubMed]

64. Zhang, F.F.; Liu, J.; Rehm, C.; Wilde, P.; Mande, J.R.; Mozaffarian, D. Trends and Disparities in Diet Quality among US Adults by Supplemental Nutrition Assistance Program Participation Status. JAMA Netw. Open 2018, 1, e180237. [CrossRef]

65. Gosliner, W. School-Level Factors Associated with Increased Fruit and Vegetable Consumption Among Students in California Middle and High Schools. J. Sch. Health 2014, 84, 559-568. [CrossRef]

66. Todd, J.E. Revisiting the Supplemental Nutrition Assistance Program cycle of food intake: Investigating heterogeneity, diet quality, and a large boost in benefit amounts. Appl. Econ. Perspect. Policy 2014, 37, 437-458. [CrossRef]

67. Nord, M.; Golla, A.M. Does SNAP Decrease Food Insecurity. Untangling the Self-Selection Effect; U.S. Department of Agriculture, Economic Research Service: Washington, DC, USA, 2009.

68. Kohn, M.J.; Bell, J.F.; Grow, H.M.G.; Chan, G. Food insecurity, food assistance and weight status in US youth: New evidence from NHANES 2007-08. Pediatr. Obes. 2014, 9, 155-166. [CrossRef]

69. Edelstein, S.; Pergamit, M.R.; Ratcliffe, C. Characteristics of Families Receiving Multiple Public Benefits; Urban Institute: Washington, DC, USA, 2014; Available online: https:/ / www.urban.org/research/publication/characteristics-families-receiving-multiplepublic-benefits (accessed on 9 August 2021).

70. Hanson, K.L.; Olson, C.M. School Meals Participation and Weekday Dietary Quality Were Associated after Controlling for Weekend Eating among U.S. School Children Aged 6 to 17 Years. J. Nutr. 2013, 143, 714-721. [CrossRef]

71. USDA Food and Nutrition Service. Final Rule: Nutrition Standards in the National School Lunch and School Breakfast Programs. Available online: https:/ / www.fns.usda.gov/cn/fr-012612 (accessed on 28 September 2021).

72. USDA Food and Nutrition Service. Nutrition Standards for School Meals. Available online: https://www.fns.usda.gov/cn/ nutrition-standards-school-meals (accessed on 28 September 2021).

73. Spronk, I.; Kullen, C.; Burdon, C.; O'Connor, H. Relationship between nutrition knowledge and dietary intake. Br. J. Nutr. 2014, 111, 1713-1726. [CrossRef]

74. Wardle, J.; Parmenter, K.; Waller, J. Nutrition knowledge and food intake. Appetite 2000, 34, 269-275. [CrossRef] [PubMed]

75. Beydoun, M.A.; Wang, Y. Do nutrition knowledge and beliefs modify the association of socio-economic factors and diet quality among US adults? Prev. Med. 2008, 46, 145-153. [CrossRef] [PubMed] 